\title{
10. A Meta-methodology for Collaborative Network Organisations: A Case Study and Reflections
}

\author{
Ovidiu Noran \\ School of Computing and Information Technology, Griffith University \\ E-Mail:O.Noran@Griffith.edu.au
}

\begin{abstract}
Presently, there is a great need for methodologies and reference models to assist and guide the creation and operation of various types of Collaborative Networked Organisations (CNO). The efforts to fulfil this need can be greatly assisted by a meta-methodology integrating diverse CNO creation and operation knowledge. This paper contimues previous research on the concept, requirements, design, verification and potential implementations of a CNO life cycle meta-methodology, by describing an additional case study and subsequent reflections leading to the refinement and extension of the proposed meta-methodology.
\end{abstract}

\section{INTRODUCTION}

In the current global market conditions, organizations worldwide often need to come together in order to bid for, and execute projects whose requirements go beyond their individual competencies. Collaborative Networked Organisations (CNO) in their various forms of manifestation (such as Virtual Organisations (VO) or Professional Virtual Communities) are recognized to offer an advantage in a competitive situation by capitalising on the overall pool of knowledge existing in the participants. However, CNO set-up and operation includes human aspects, such as establishing partner trust and a sense of community, which can only be effectively addressed in time. Similarly, CNO technical aspects such as the establishment of agreed business practices, common interoperability and distributed collaboration infrastructures require time that may not be always available. 'Business ecosystems', 'breeding / nesting environments' (Camarinha-Matos, 2002), or 'company networks' (Globemen, 2000-2002) may enable a prompt formation of CNOs; however, they need to be supported by effective reference models containing methods describing CNO set-up and operation. Unfortunately, the methodologies contained in such reference models are too generic to allow their effective use for a particular project; thus, specific methodologies have to be created for each scenario. In this sense, a meta-methodology ${ }^{47}$ may be of great help in integrating knowledge relating to several CNO-type creation and operation methodologies, and thus being able to promptly suggest a suitable method for a particular $\mathrm{CNO}$ project.

This paper presents the application of the proposed meta-methodology to a specific scenario: the creation of a VO in the higher education sector. In this context,

\footnotetext{
${ }^{47}$ a method (here, consisting of steps and their applicability rules) on how to design a method.
} 
the meta-methodology is used both in practice (to create a VO formation / operation method for the participating organisations) and in theory (for the triangulation ${ }^{48}$ of the meta-methodology concept and for reflections leading to its extension), thus fulfilling the dual purpose of action research (McKay \& Marshall, 2001).

\section{META-METHODOLOGY PRIMER}

\subsection{Research Question and Strategy}

The research question has asked whether a methodology describing how to construct customised modelling methods may be built and what other factors may (positively) influence such an endeavour. This topic has provided an opportunity to employ action research (Galliers, 1992; Wood-Harper, 1985), which allows for both practical problem solving and generating / testing theory (Eden \& Chisholm, 1993; McKay \& Marshall, 2001). The research strategy was based on two cycles containing lab / field testing and reflections leading to theory extension (Checkland, 1991). The cycles are entered after stating the research question, completing the research design and adopting the theoretical model. The cycles' exit conditions are based on a compromise between the time / resources available and the accuracy of the result. The design of the research strategy (design decisions, research stance justifications and methods adopted) is described in (Noran, 2001), while conceptual development of the meta-methodology and testing are contained in (Noran, 2004c).

\subsection{A First Case Study}

In this case study, the application of the meta-methodology has yielded a design (and partly operation) method for a Breeding Environment $(\mathrm{BE})^{49}$ and the Service Virtual Enterprises (SVEs) created by the BE (Hartel et al., 2002). The lead partner(s) ${ }^{50}$ wished to retain control of the identification and the concept of the SVEs created, with the rest of the SVEs' life cycle phases covered by the BE. The model audience (various levels of management and technical personnel) was partly familiar with the IDEF $^{51}$ family of languages and with the Globemen ${ }^{52}$ reference model.

The application of the meta-methodology has resulted in a multi-level IDEF $0^{53}$ model of the design methodology for the BE and the SVEs created by it, based on the Globemen Reference Model (due to audience proficiency). The chosen language has allowed different levels of the model to simultaneously target various audiences (management, working groups, etc), while ensuring the overall model consistency. The methodology has received a positive response and is being currently used in BE creation and operation, and in SVE creation.

\footnotetext{
48 triangulation is possible in the context of an previous case study (Bernus et al., 2002) and based on the assumption that while humans subjectively interpret reality, it is possible to build a descriptive and commonly agreed upon methodology model (Noran, 2004c).

49 called Service Network Organisation (SNO) in this case study

${ }^{50}$ one or several partners that initiate BE creation and may influence SVEs created by the BE.

${ }^{51}$ Integration DEFinition, a family of languages aiming to create computer-implementable modelling methods for analysis and design (Menzel \& Mayer, 1998).

52 Global Engineering and Manufacturing in Enterprise Networks (Globemen, 2000-2002)

${ }^{53}$ Integration Definition for Function Modelling (NIST, 1993) - an IDEF functional language
} 
The meta-methodology has also recommended modelling of the decisional aspect of the partners, BE and potential SVE(s) using GRAI54 Grids and applicable reference models55. This case study is described in detail in (Bernus et al., 2002).

\subsection{Meta-methodology Content Before the Second Case Study}

In brief, before the second case study the meta-methodology comprised the following steps and associated rules of application:

- identify enterprise entities involved in the BE / CNO creation task: mandatory;

- create a business model able to express relations between life cycle phases of the identified enterprise entities (using a suitable formalism): mandatory;

- create an activity model of the $\mathrm{BE} / \mathrm{CNO}$ design and operation: mandatory (main deliverable), depth level according to requirements;

- recommend additional aspects to be modelled and formalisms / tools (such as information, decision, organisation, time): project specific .

\section{A SECOND CASE STUDY}

This case study has been chosen due to significant differences from the first case study regarding the participant organisations' type, culture and the target CNO. This noticeable disparity has enabled the effective testing, triangulation and reflection leading to the extension of the proposed meta-methodology.

\subsection{Background}

Faculty FAC within university U contains several schools (A to D), with schools A and $B$ having the same profile. School A is based in two campuses, situated at locations L1 and L2, while school B is based on single campus, situated at location L3 (AS-IS in Figure 4). Although of the same profile, and belonging to the same FAC and U, schools A and B are confronted with a lack of consistency in their products and resources, such as the programs and courses offered, allocated budget, research higher degree (RHD) scholarship number and conditions, academic profile and availability of teaching staff, etc. This situation causes negative effects, such as additional costs and difficulty in student administration and course / program design / maintenance, inter-campus competition for RHD students and staff fallacies of unequal academic and professional standing between campuses, all of which are detrimental to the Faculty as a whole.

\section{Proposed Solution}

The issues previously described could be optimally resolved by schools A and B forming a VO (called merged school (MS) in the TO-BE state in Figure 4) with cross-campus management and policies ensuring intrinsic consistency in the product delivered and resources allocated to the individual campuses at L1, L2 and L3. Thus, the individual campuses are set to retain much of their internal decisional and organisational structure except for the highest layer, which will be replaced by the

\footnotetext{
${ }^{54}$ Graphes avec Résultats et Activitées Interreliées (Graphs with Results and Activities Interrelated), a decisional modelling formalism / reference model (Doumeingts et al., 1998) ${ }^{55}$ such as the Partner-BE-SVE reference model described in (Olegario \& Bernus, 2003)
} 
VO governance structure. Campus interoperability within the VO will be assisted by a common ICT infrastructure (Camarinha-Matos \& Afsarmanesh, 1999) ${ }^{56}$.

\subsection{Specific Features of the VO Formation Scenario}

In this case study, the function of Breeding Environment (BE) internal to $U$ could be performed by the Faculty FAC, which contains several schools forming VOs as necessary. The largest school participating in an internal VO formation project (e.g. school A in Figure 2 or Figure 4) could be identified as the lead partner. In the current situation, partners $\mathrm{A}$ and $\mathrm{B}$ within the $\mathrm{BE}$ have come together at the initiative of the $\mathrm{BE}$ and the lead partner for the purpose of an on-going VO project; thus, the VO was set to have a long life, possibly equal to that of the BE (the FACulty). Importantly, the partners will cease to operate independently during the life of MS.

Note that, in the internal environment (U), the frequency of VO creation and thus the degree of preparedness for VO creation of the partners (schools) within the BEs (Faculties) in U, is low. However, it is likely that individual, or groups of schools will increasingly participate in BEs and VOs outside $\mathrm{U}$, in which case additional agility (Goranson, 1999) and preparedness is required. This could be achieved by using reference models (reusable templates) of possible VO types, which should include VO set-up and operation methodology templates. However, the use of such reference models requires user proficiency and implies further customisation work. Thus, the proposed meta-methodology can assist in enhancing preparedness and agility by producing a methodology suited to a specific VO creation / operation task

The audience of the deliverables was to be the management of U, FAC, A and B and the academic and general staff of $\mathrm{A}$ and $\mathrm{B}$. This audience diversity dictated that the chosen formalisms should allow effective complexity management, and that primers to the modelling methods must be included with the model(s). This has led to the choice of IDEF0 language for functional, and GRAI Grids for decisional / organisational57 modelling. A simplified version of rich pictures (Checkland \& Scholes, 1990) have also been considered, in order to facilitate a common understanding of the models. In addition, it was desired that the chosen modelling tool(s) be capable of versioning and Internet publishing in order to facilitate model management and dissemination.

Documentation from a previous similar project was available, although its particular nature required additional effort for its use as a possible reference mode ${ }^{58}$.

\subsection{Meta-methodology Application}

The case study has been approached using the meta-methodology content described in section 2.3. As expected, the practical application of the meta-methodology to a markedly different situation has brought about some step variations and additions, leading to the enrichment and refinement of the meta-methodology.

\footnotetext{
${ }^{56}$ this requirement is partially satisfied initially, because all campuses belong to the same University $U$ that owns (or leases) the cross-campus infrastructure.

${ }^{57}$ may be represented in a GRAI-Grid by assigning human resources to decision centres.

58 e.g. filtering out noise and abstracting reusable knowledge from the available information.
} 


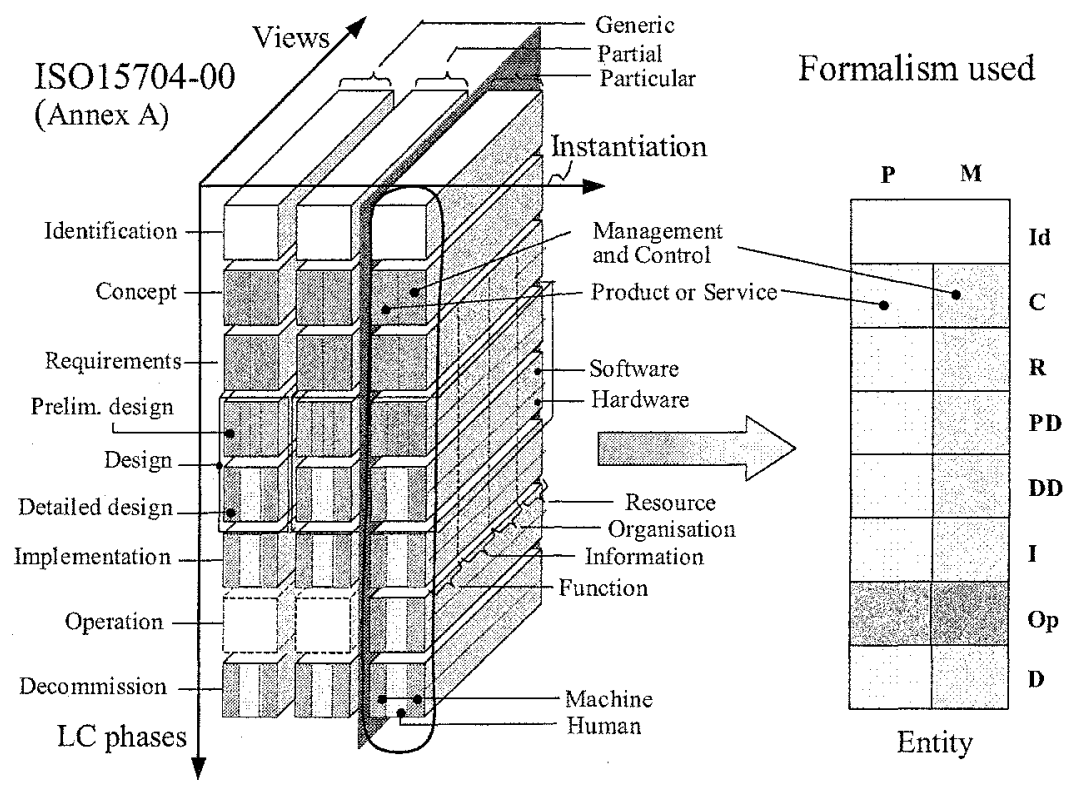

Figure 1. Model used: simplified ISO15704 Annex A

\section{Step One: Identification of the Entities Involved.}

This step has included a) deciding on the entity aspects that need to be modelled, b) the choice of a suitable entity modelling formalism, expressive enough to cover aspects decided in a), and c) constructing an initial ${ }^{59}$ set of entities relevant to the project, in a form usable for the next step. In addition, the meta-methodology required a modelling formalism able to represent the selected entity aspects in the context of the entity life cycle, and capable to express the interaction of life cycle phases both within the same entity and between separate entities.

The modelling framework of ISO 15704 Annex A (GERAM ${ }^{50}$ ), namely the Generalised Enterprise Reference Architecture (GERA) contains placeholders for concepts such as life cycle phases and views; they can be used in selecting suitable architecture frameworks to create life cycle models of various entity aspects ${ }^{61}$ at various levels of abstraction ${ }^{62}$.

Using GERA as a checklist of possible views in this particular case, it has been initially established that the main aspects needed to be modelled during the life of the entities involved were the management, and the product / service. Subsequently, it has become clear that decisional and organisational models of the entities directly participating in the VO formation will also be necessary.

\footnotetext{
${ }^{59}$ this set may be subsequently revised as further described

${ }^{60}$ Generalised Enterprise Reference Architecture and Methodology (ISO/TC184, 2000)

${ }^{61}$ this capability is inherited from the architecture frameworks GERAM originated from, i.e. the Purdue Enterprise Reference Architecture (PERA) (Williams, 1994), the Open System Architecture for CIM (CIMOSA Association, 1996) and GRAI-GIM (Doumeingts, 1984) ${ }^{62}$ GERA may also be used to guide the creation of partial models, glossaries, meta-models and ontologies
} 
Constructing a useful set of entities requires knowledge of the participating entities; if not already possessing it, the meta-methodology user needs to acquire it by reviewing relevant documentation, interviewing stakeholders and even temporarily entering the organisations participating in the VO formation .

\section{Step Two: A Business Model expressing Life Cycle Relations}

The construction of the business model has included refinements of the entity set through additional iterations of the step one. Entities whose life cycle was found not to be highly relevant to the model have been collapsed into a single phase (usually operation); conversely, entities which initially had only been modelled in their operation phase but whose various life cycle phases have become of interest during this step, have had their representation expanded to include their entire life cycle. The scope of the refinement is to improve the accuracy of the Business Model, and thus of the resulting method.

The business model (shown in Figure 2) describes the relations between life cycle phases of the entities identified in step one. As can be seen, several entities influence various life cycle phases of the VO entity, directly, or through other entities' life cycle phases. By 'reading' the life cycle diagram of MS, one can infer activities necessary at each life cycle phase for the creation of the VO and elicit the entities involved in these activities, as described in step four below.

Once again, an accurate business model demands deep knowledge of the structures and relations between participating entities. Generally, the business model may be constructed either to reflect a combined AS-IS / TO-BE view (such as used in this case study - see Figure 2), or it can be split in sub-models for the present and desired state(s); the choice of representation depends on model complexity and on the audience preferences. Once the model is constructed, it needs to be submitted for review and feedback to the stakeholders in order to improve its accuracy and achieve acceptance. The quality of the business model is paramount to the creation of an effective VO creation / operation method.

\section{Step Three (Additional): Models of the AS-IS and TO-BE States}

The target VO was envisaged to reuse as much as possible from the existing entities' structures and thus not to differ radically from the existing individual entities, except for the very top governance layer. In addition, there was a need to fully understand the existing entities' operation and to decide on a decisional and organisational structure of the VO. Thus, it has become necessary to undertake an additional step within the meta-methodology application, i.e. to construct models of the decisional and organisational aspects for the AS-IS ${ }^{63}$, and several proposed TO-BE states.

The AS-IS model has revealed a large degree of intervention of the Planning decision centre in both the Product and the Resources decision centre groups at most horizons. This corresponds to a high degree of turbulence and a lack of clear and effective strategy within the organisation. In essence, the Head of School (HOS) in the role of Planner has to put out 'fires' (product/ resource discrepancies requiring immediate reconciliation) on a short-term basis, rather than having strategies in place to avoid the cause of such problems. The AS-IS decisional model has also

${ }^{63}$ AS-IS modelling is beneficial if management believes that the desired TO-BE state may be achieved by improving the AS-IS state rather than replacing it (Uppington \& Bernus, 2003) 
shown a lack of sufficient financial and decisional independence of the schools A and $B$ and a shortage of information crucial to long-term strategy making ${ }^{64}$.

Thus, in constructing the TO-BE decisional model, attention has been paid to confine the authoritarian role of Planning to the strategic level ${ }^{65}$, to increase the financial and decisional independence of the target VO (MS), and to provide the necessary external information to MS for the purpose of strategy making and selfgovernance. The strategy thus created can then be used by lower decision centres to set decision frameworks for subordinate centres in a top-down (rather than lateral) fashion, resulting in more predictable organisational behaviour and less turbulence.

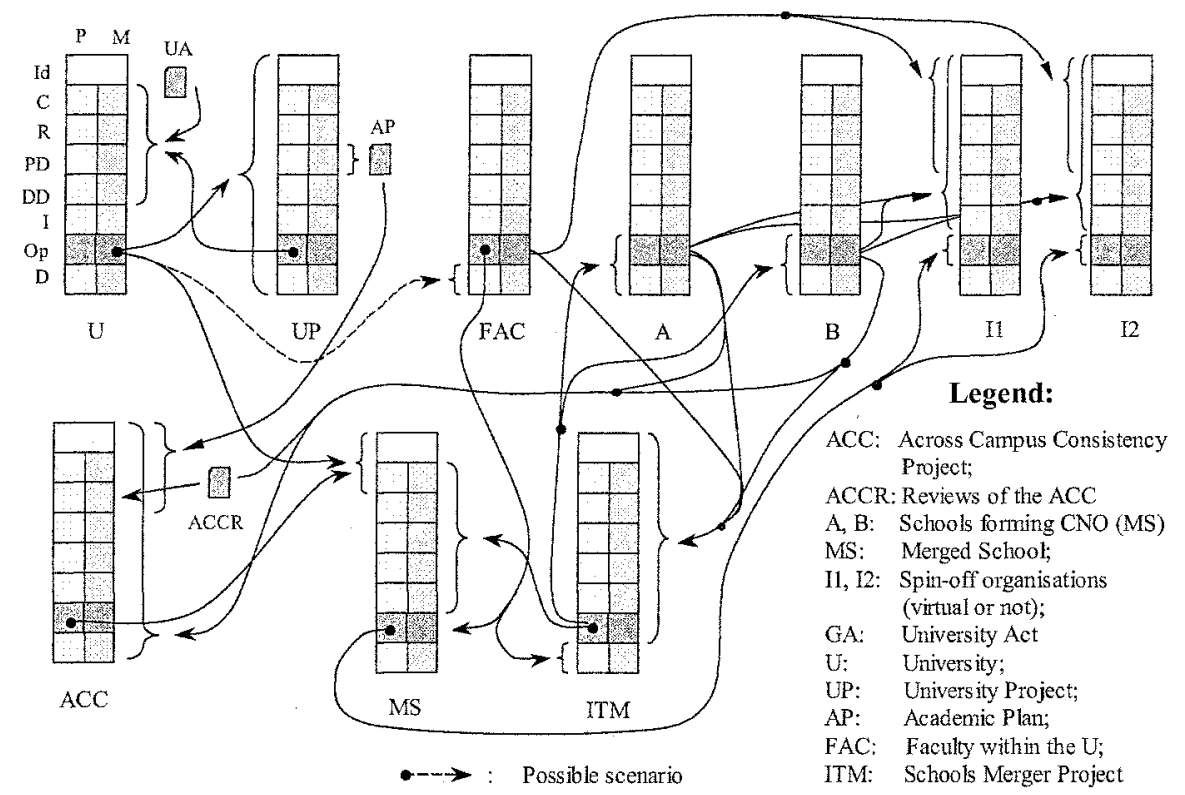

Figure 2 Business model expressing entity life cycle phases' relations

Interestingly, the resulting TO-BE decisional model has been found to match all envisaged scenarios, with differences only obvious in the organisational structure, i.e. the allocation of the various human resource groups to the decision centres represented in a single TO-BE decisional framework (Figure 3). Thus, the various scenarios were based on the same decisional framework, while proposing various decisional roles and memberships of these roles. For example, all scenarios agreed on the existence of a School Executive and its allocation to approximately the same decision centres, but differed on the composition of that body (e.g. from one person (HOS) to several (HOS plus Associate Heads of Schools)).

${ }^{64}$ e.g. the lack of information regarding generated income and projected size of allocated budget over several years means that the frequency of budget allocation from higher echelons (e.g. yearly) directly constrains the horizon over which effective strategy can be made

65 thus, highly demanded resources presently allocated to Planning at lower levels (e.g. HOS, Executive) may be redistributed at higher levels for greater efficiency 


\section{Sub-step: Additional Representations}

The type and cultures of the participating organisations have brought about the need for broad consultation and feedback. The diverse audience background and limited time available has necessitated the use of an additional sub-step, namely the use of rich pictures (Figure 4). They have allowed to promptly and effectively communicate the stakeholders' vision on the future VO options and have also acted as a primer to the complete, but more complex representations shown in Figure 3.

\section{Step Four: Activity Model of the VO Design and Operation}

The creation of the Activity model started by 'reading' the life cycle diagram of the VO to be designed and its relations with the other entities, as shown in Figure 2. The set of activities obtained was then recursively refined and decomposed into subactivities, until reaching a level of detail deemed sufficient to control specific VO creation tasks. The model had to be regularly submitted to the stakeholders for consultation and validation, thus ensuring continuing management support. In addition, the model was also presented for comments to the various working groups, for feedback and to obtain commitment towards the actual use of the model. The chosen modelling language (IDEF0) has allowed to develop an integrated set of diagrams on various levels of detail, which have allowed the Working Groups (WG) involved in the VO creation to operate in parallel within a consistent framework.

Figure 5 describes the second level (A0 diagram) of the IDEF0 activity model describing VO design and operation. The modelling tool used was $\mathrm{KBSI}^{66} \mathrm{AIOWin}$, which is web enabled and integrated with information / resources modelling (SmartER) and behaviour / simulation modelling (ProSIM) tools by the same vendors, thus ensuring model(s) consistency ${ }^{67}$.

A detailed description of the activity model creation and structure (which is beyond the purpose of this paper) is contained in (Noran, 2004b).

\section{Step Five: Other Aspects to be Modelled}

Due to the particulars of the organisations involved in the case study, the metamethodology has identified several additional aspects to be modelled, such as:

- Organisational culture and design: e.g. gap analysis, gap reduction strategies for the organisations participating in the VO, change management strategies;

- A time dimension for the set of activities in the VO creation project;

- AS-IS / TO-BE comparative costs to the University

- IS / IT infrastructure associated with production and customer service.

Modelling of such aspects must employ relevant available reference models whenever possible. For example, the first aspect has used (Keidel, 1995) to identify the organisational pattern of the schools involved, and has used gap analysis concepts from (Ansoff, 1965; Howe, 1986) and the (Kotter, 1996) eight-stage organisational change process.

\footnotetext{
${ }^{66}$ Knowledge-Based Systems, Inc. www.kbsi.com

${ }^{67}$ the IDEF family of languages is presently not integrated by a published metamodel (Noran, 2003 ); thus, consistency across models must be enforced by the modelling tools or the user.
} 
The resulting method and its by-products have been well received, allowing working groups to refine their concepts, to develop and communicate several models and to use an ordered and consistent activity set throughout the VO creation project.

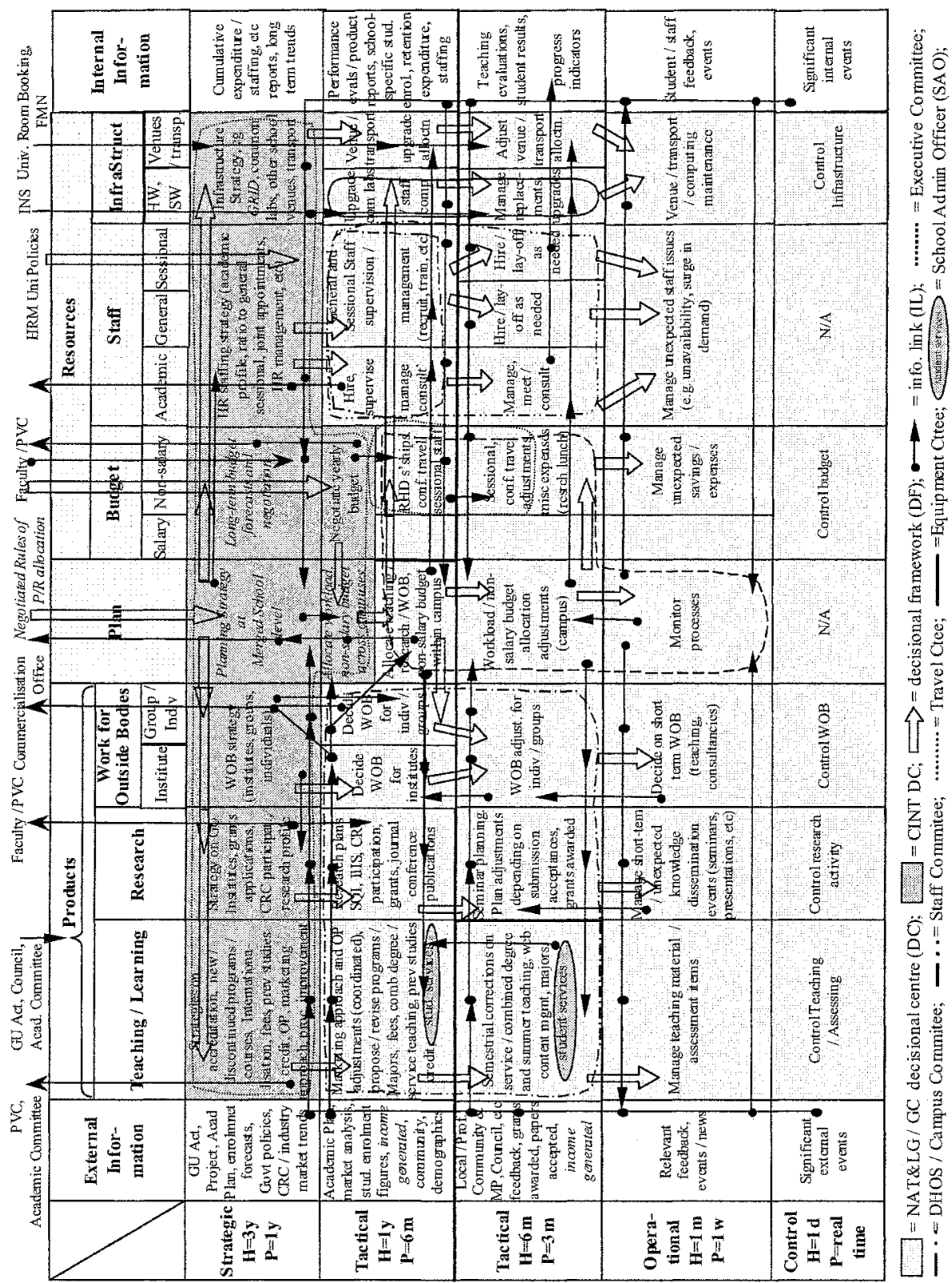

Figure 3. To-BE Decisional Model (Noran, 2004a) 


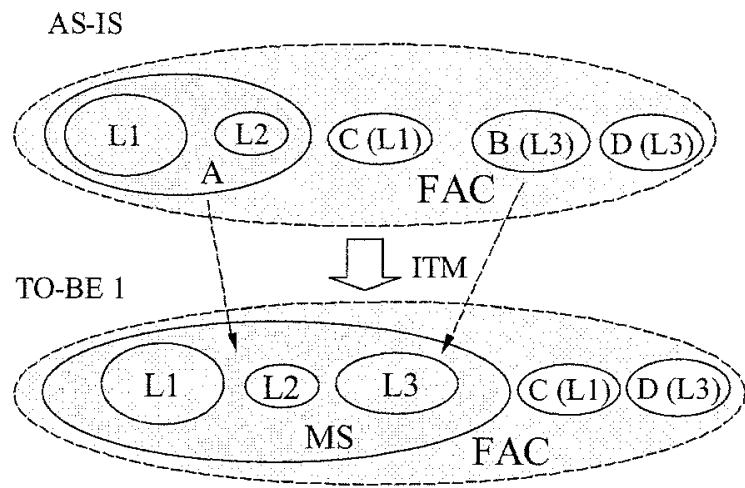

Legend:

FAC: Faculty (BE?)

A...D: schools within FAC

L1.. L3: physical locations

ITM: Schools merger project

MS: Merged School (VO)

HOS: Head of School

DHOS: Deputy HOS

PA: Personal Assistant

SAO: School Admin Officer

GS: number of General Staff (not all staff shown)

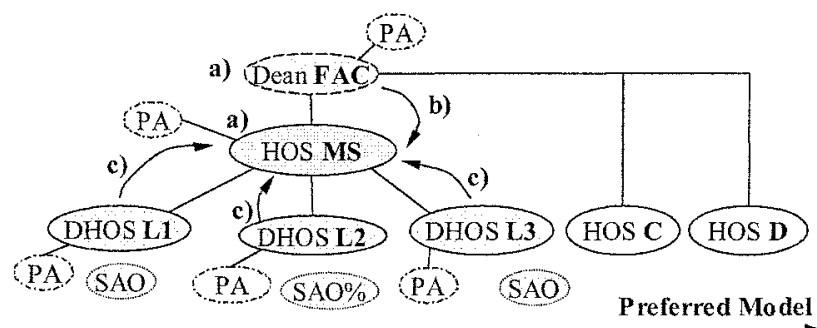

\section{Organisation:}

One FAC Dean, one HOS of MS and a DHOS on each MS campus

a) Dean FAC and HOS MS are distinet $(\mathrm{GS}=7.5)$

b) Dean FAC is also HOS MS (GS=6.5)

c) HOS MS is one of the DHOS, nominated by rotation or ProVice Chancelor (GS $=6.5$ )

Figure 4. Rich picture of the AS-IS and of a possible TO-BE state (Noran, 2004a)

\section{TRIANGULATION AND REFLECTION}

The different nature of the case studies has allowed an effective triangulation of the feasibility of the meta-methodology concept and its content - i.e. the set of steps and application rules. Thus, in two different cases it has been found that the methods produced by the meta-methodology have helped and effectively guided the creation and operation of BEs and CNOs.

In addition, as expected and desired, the second case study has enabled reflection leading to the refinement and extension of the meta-methodology. This extension is best expressed in the revised set of steps and their application descriptions below:

- Entity identification should be performed in all cases. It is now clear that the chosen modelling formalism should be able to express life cycle phases (for step two). The formalism needs to be restricted to the aspects relevant to the task at hand. For example, both case studies have used a simplified GERA, showing only life cycle phases and the Management / Control vs. Production aspects;

- The business model containing the entities' life cycles phases and their relations enables the production of the main meta-methodology deliverable, and as such it should always be constructed. The business model may be represented in separate, or in combined AS-IS /TO-BE diagrams, depending on the model complexity and audience proficiency / preference; 


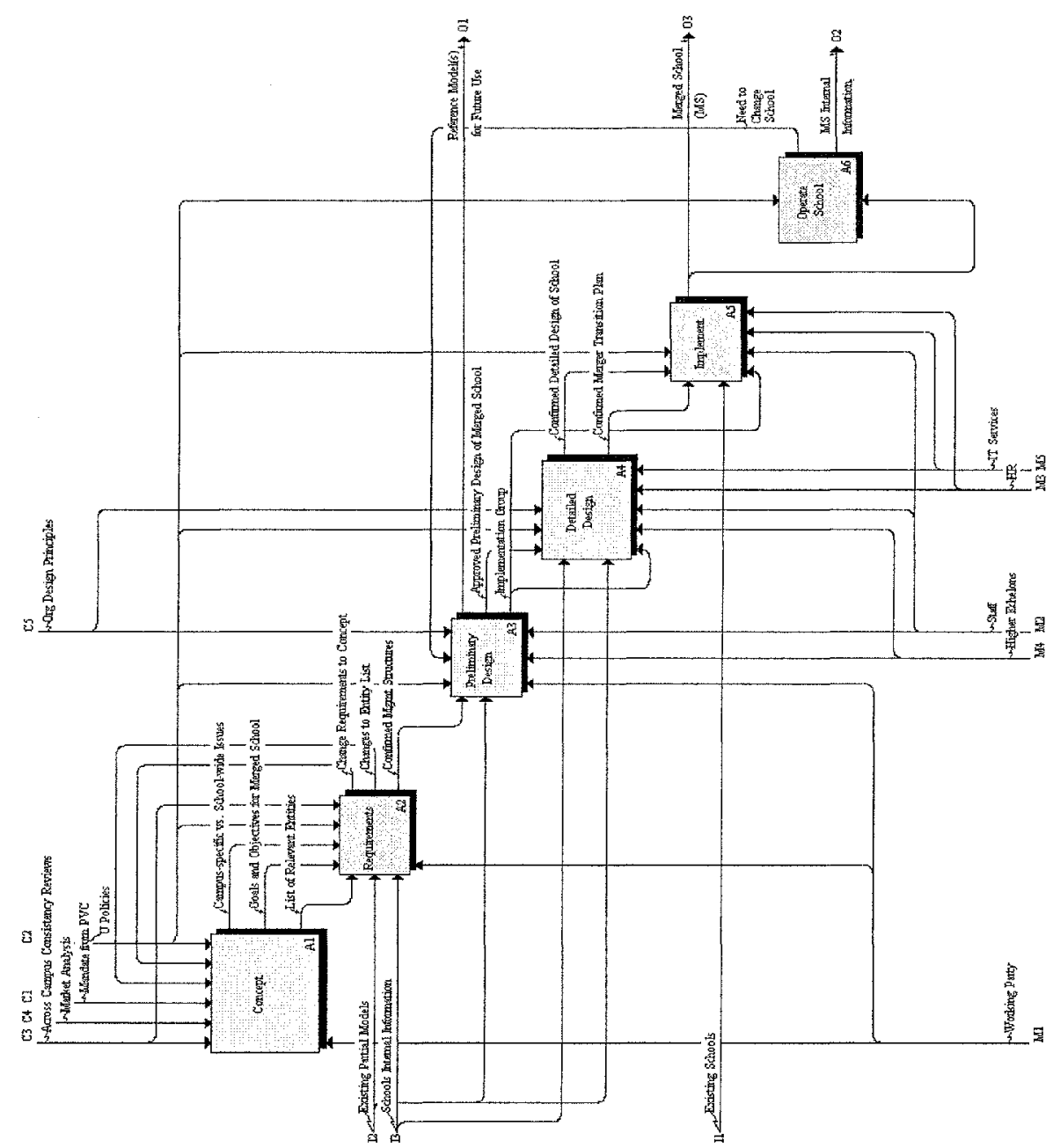

Figure 5 IDEF0 model of the merger method

- Modelling the present (AS-IS) state of the participating organisations is needed especially if it is not fully understood by stakeholders or if the future state can be obtained by evolving rather than replacing the AS-IS. Modelling of the future (TO-BE) state(s) is needed especially if the stakeholders have not yet agreed on the structure of the future organisation. AS-IS and TO-BE models may be required for several aspects, such as functional (and decisional), informational, organisational, resources, ete ${ }^{68}$. A modelling formalism is to be chosen based on audience proficiency and suitability and a primer to the chosen formalisms has to be provided. Additional representations may also be needed to assist understanding by the stakeholders and to communicate their vision to the staff;

${ }^{68}$ for example, if organisational design is needed, then modelling of the TO-BE decisional and organisational states has to be performed 
- Step four of the meta-methodology produces the main meta-methodology deliverable (the VO creation method) and hence is mandatory. This step may occur concurrently with the step three, if the latter is at all performed;

- Some additional aspects recommended to be modelled (step five) are initially known to be of importance to the project, while others may emerge during method application.

The application of the meta-methodology steps in relation to the target VO creation project (ITM) life cycle is shown in Figure 6 . Note that it is possible that the VO creation / operation method may start being applied before it is fully completed.

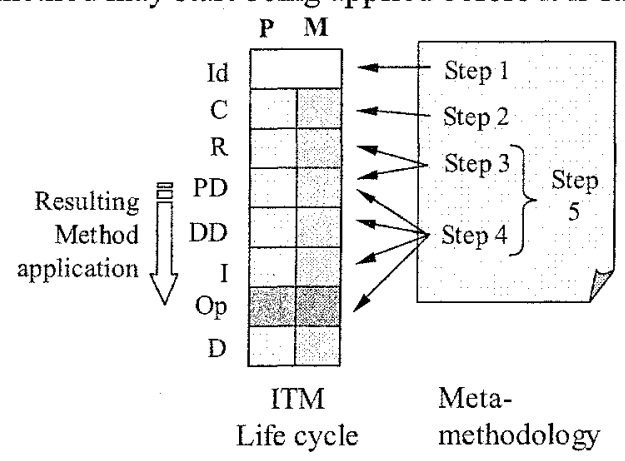

Figure 6. Application of the meta-methodology steps in relation to ITM life cycle

\section{CONCLUSION AND FURTHER WORK}

This paper has presented the application of the proposed meta-methodology within a second case study, which has enabled the triangulation of the proposed concept and reflections resulting in additions and refinements of the meta-methodology steps.

The main conclusion is that it is possible to construct a method (i.e. a set of steps with associated applicability rules) on how to produce customised VO creation / operation sets of activities and supporting by-products for specific scenarios.

The meta-methodology created in the attempt to answer the research question proves the feasibility of the concept, however it is by no means complete. Further refinement can be achieved by its application to a significant number of case studies and by incorporating $\mathrm{BE}, \mathrm{CNO}$ and $\mathrm{VO}$ creation and operation knowledge from various streams of research. The open character of the meta-methodology allows its extension subject to some basic constraints, such as avoiding contradicting the existing rules (e.g. by performing a reconciliation of the existing and proposed steps $/$ application rules, or by creating profiles for project categories) or preventing knowledge scattering over an excessive amount of steps and application rules.

The space and scope limitations of this paper do not allow the in-depth presentation of the meta-methodology; however, a full description of the findings and contributions are described in (Noran, 2004b).

\section{REFERENCES}

Ansoff, H. I. (1965). Corporate Strategy. New York: McGraw-Hill.

Bernus, P., Noran, O. and Riedlinger, J. (2002). Using the Globemen Reference

Model for Virtual Enterprise Design in After Sales Service. In I. Karvoinen et al. 
(Eds.), Global Engineering and Manufacturing in Enterprise Networks (Globemen) VTT Symposium 224. Helsinki / Finland. 71-90.

Camarinha-Matos, L. M. (2002). Foreword. In L. M. Camarinha-Matos (Ed.), Collaborative Business Ecosystems and Virtual Enterprises (Proceedings of PROVE02: 3rd IFIP Working Conference on Infrastructures for Virtual Enterprises) (Sesimbra/ Portugal: Kluwer Academic Publishers.

Camarinha-Matos, L. M. and Afsarmanesh, H. (1999). Infrastructures for Virtual Enterprises: Kluwer Academic Publishers.

Checkland, P. (1991). From Framework through Experience to Learning: the Essential Nature of Action Research. In H.-E. Nissen, et al. (Eds.), Information Systems Research: Contemporary Approaches \& Emergent Traditions. Amsterdam: Elsevier.

Checkland, P. and Scholes, J. (1990). Soft Systems Methodology in Action. New York: J. Wiley \& Sons.

CIMOSA Association. (1996). CIMOSA - Open System Architecture for CIM,. Technical Baseline, ver 3.2. Private Publication.

Doumeingts, G. (1984). La Methode GRAI (PhD Thesis). Bordeaux, France: University of Bordeaux I.

Doumeingts, G., Vallespir, B. and Chen, D. (1998). GRAI Grid Decisional Modelling. In P. Bernus et al. (Eds.), Handbook on Architectures of Information Systems. Heidelberg: Springer Verlag. 313-339.

Eden, M. and Chisholm, R. F. (1993). Emerging Varieties of Action Research: Introduction to the Special Issue. Human Relations, 46, pp. 121-142.

Galliers, R. D. (1992). Choosing Information Systems Research Approaches. In R. Galliers (Ed.), Information Systems Research - Issues, Methods and Practical Guidelines. A.Waller Ltd. 144-162.

Globemen. (2000-2002). Global Engineering and Manufacturing in Enterprise Networks. IMS project no. 99004 / IST-1999-60002. Available: http://globemen.vtt.fi/.

Goranson, H. T. (1999). The Agile Virtual Enterprise. Westport: Quorum Books.

Hartel, I., Billinger, S., Burger, G. and Kamio, Y. (2002). Virtual Organisation of the After-sales Service in the One-of-a-kind Industry. In L. Camarinha-Matos (Ed.), Collaborative Business Ecosystems and Virtual Enterprises (Proceedings of PROVE02: 3rd IFIP Working Conference on Infrastructures for Virtual Enterprises). Sesimbra / Portugal. pp. 405-420.

Howe, W. S. (1986). Corporate Strategy. London: MacMillan Education.

ISO/TC184. (2000). Annex A: GERAM. In ISO-2000 (Ed.), ISO/IS 15704: Industrial automation systems - Requirements for enterprise-reference architectures and methodologies.

Keidel, R. W. (1995). Seeing Organizational Patterns: a New Theory and Language of Organizational Design. San Francisco: Berrett-Koehler Publishers.

Kotter, J. P. (1996). Leading Change. Boston, MA: Harvard Business School Press.

McKay, J. and Marshall, P. (2001). The Dual Imperatives of Action Research. Information Technology \& People, 14(1), pp. 46-59.

Menzel, C. and Mayer, R. J. (1998). The IDEF Family of Languages. In P. Bernus et al. (Eds.), Handbook on Architectures of Information Systems. Heidelberg: Springer Verlag Berlin. pp. 209-241. 
NIST. (1993). Integration Definition for Function Modelling (IDEF0) (Federal Information Processing Standards Publication 183): Computer Systems Laboratory, National Institute of Standards and Technology.

Noran, O. (2001). Research Design for the PhD Thesis: Enterprise Reference Architectures and Modelling Frameworks, (Report / Slides). School of CIT, Griffith University. Available: www.cit.gu.edu.au/ noran [2001, 2001]. Noran, O. (2003, Dec 2003). UML vs. IDEF: An Ontology-oriented Comparative Study in View of Business Modelling. In Proceedings of 6th International Conference on Enterprise Information Systems (ICEIS 2004), Porto (Portugal).

Noran, O. (2004a). Application of the Meta-methodology for Collaborative Networked Organisations to a University School Merger, [Report to the IT School Merger Project Working Party]. School of CIT, Griffith University.

Noran, O. (2004b). A Meta-methodology for Collaborative Network Organisations, $\mathrm{PhD}$ Thesis. School of CrT, Griffith University.

Noran, O. (2004c). Towards a Meta-methodology for Cooperative Networked Organisations. In Proc. of 5th IFIP Working Conference on Virtual Enterprises (PRO-VE04), Toulouse / France.

Olegario, C. and Bernus, P. (2003). Modelling the Management System. In P. Bernus, et al. (Eds.), Handbook on Enterprise Architecture. Heidelberg: Springer Verlag. pp. 435-500.

Uppington, G. and Bernus, P. (2003). Analysing the Present Situation and Refining Strategy. In P. Bernus et al. (Eds.), Handbook on Enterprise Architecture. Heidelberg: Springer Verlag. pp. 309-332.

Williams, T. J. (1994). The Purdue Enterprise Reference Architecture. Computers in Industry, 24(2-3), pp. 141-158.

Wood-Harper, A. T. (1985). Research methods in IS: Using Action Research. In E. Mumford, et al. (Eds.), Research Methods in Information Systems - Proceedings of the IFIP WG 8.2 Colloquium. Amsterdam: North-Holland. pp. 169-191 